\title{
Effect of electric stimulation on human chondrocytes and mesenchymal stem cells under normoxia and hypoxia
}

\author{
BETTINA HIEMER, MARTIN KROGULL, THOMAS BENDER, JOSEFIN ZIEBART, \\ SIMONE KRUEGER, RAINER BADER and ANIKA JONITZ-HEINCKE \\ Department of Orthopaedics, Biomechanics and Implant Technology Research Laboratory, \\ Rostock University Medical Centre, D-18057 Rostock, Germany
}

Received October 13, 2017; Accepted April 30, 2018

DOI: $10.3892 / \mathrm{mmr} .2018 .9174$

\begin{abstract}
During joint movement and mechanical loading, electric potentials occur within cartilage tissue guiding cell development and regeneration. Exposure of cartilage exogenous electric stimulation (ES) may imitate these endogenous electric fields and promote healing processes. Therefore, the present study investigated the influence of electric fields on human chondrocytes, mesenchymal stem cells and the co-culture of the two. Human chondrocytes isolated from articular cartilage obtained post-mortally and human mesenchymal stem cells derived from bone marrow (BM-MSCs) were seeded onto a collagen-based scaffold separately or as co-culture. Following incubation with the growth factors over 3 days, ES was performed using titanium electrodes applying an alternating electric field $(700 \mathrm{mV}, 1 \mathrm{kHz})$. Cells were exposed to an electric field over 7 days under either hypoxic or normoxic culture conditions. Following this, metabolic activity was investigated and synthesis rates of extracellular matrix proteins were analyzed. ES did not influence metabolic activity of chondrocytes or BM-MSCs. Gene expression analyses demonstrated that ES increased the expression of collagen type II mRNA and aggrecan mRNA in human chondrocytes under hypoxic culture conditions. Likewise, collagen type II synthesis was significantly increased following exposure to electric fields under hypoxia. BM-MSCs and the co-culture of chondrocytes and BM-MSCs revealed a similar though weaker response regarding the expression of cartilage matrix proteins. The electrode setup may be a valuable tool to investigate the influence of ES on human chondrocytes and BM-MSCs contributing to fundamental knowledge including future applications of ES in cartilage repair.
\end{abstract}

Correspondence to: Ms. Bettina Hiemer, Department of Orthopaedics, Biomechanics and Implant Technology Research Laboratory, Rostock University Medical Centre, 142 Doberaner Strasse, D-18057 Rostock, Germany

E-mail: bettina.hiemer@med.uni-rostock.de

Key words: human chondrocytes, mesenchymal stem cells, electric stimulation, cartilage healing

\section{Introduction}

Cartilage defects are caused by various mechanisms, such as traumatic joint damage, osteochondrosis dissecans, as well as primary and secondary degeneration. The damage of the cartilage surface influences the cartilage mechanics resulting in enhanced wear rates, leading to osteoarthritis (OA). Catabolic events during the process of OA reduce the extracellular osmolarity, which results in decreased viscoelasticity of the tissue and inferior biomechanical function (1). Because of the missing intrinsic regeneration capacity, the degeneration of the cartilage proceeds and causes severe pain for the patient (2). No successful therapy is currently available which can stop or reverse the progression of OA. Current therapy approaches, including medication, analgesics and total joint replacement provide only palliative treatment (3). Therefore, it is necessary to find new therapeutic approaches to support the regeneration of hyaline cartilage.

A common repair technique for cartilage reconstruction is the matrix-associated autologous chondrocyte implantation (MACI). This two-step procedure includes the isolation of autologous chondrocytes from a minor load-bearing area of patient's hyaline articular cartilage, followed by cell expansion in vitro and cell transfer onto a biomaterial for transplantation into the defect. A variety of different biomaterials (synthetic and natural) have been tested with respect to their application for cartilage repair. Collagen as the common protein in the cartilage tissue has been widely studied for cartilage regeneration. In particular, collagen supports cell attachment, proliferation, migration and differentiation (4,5). Studies had shown that collagen type I-based biomaterials support the chondrogenic differentiation of rat bone marrow-derived mesenchymal stem cells (BM-MSCs) and increase production of extracellular matrix in human chondrocytes $(\mathrm{CH})(6,7)$.

However, two-dimensional (2D) environment and the cell expansion in vitro results in de-differentiation of $\mathrm{CHs}$ to a fibroblast-like cell phenotype. This is characterized by decreased collagen type II synthesis and increased collagen type I expression rates $(8,9)$. The implantation of de-differentiated CHs could result in formation of fibrocartilage which is not able to withstand the high biomechanical loading in the knee joint $(10,11)$. Therefore, de-differentiated cells have to be re-differentiated (by e.g., 3D cultures) to restore the defect with 
hyaline-like tissue. In various studies, BM-MSCs are expected to be a useful cell source for cartilage tissue engineering due to the ease of harvest and the high differentiation potential of BM-MSCs (12-14). Especially, the co-cultivation of both cell types results in a robust chondrogenic differentiation which is probably triggered by signaling via cell-cell contacts and secreted factors generated by both cell types (15-17). However, to create functional cartilage tissue for implantation, specific techniques are required to facilitate adequate chondrogenesis during in vitro cultivation (18). Besides adapting the cultivation conditions to the physiological in vivo situation, providing a cartilage-like oxygen environment or the use of essential growth factors and cytokines, biophysical stimulation could be a promising approach to achieve hyaline-like cartilage formation (1).

As in bone tissue, mechanoelectric transduction occurs naturally within the cartilage. During weight-bearing and joint movement, the fluid flow over fixed ionized macromolecules within the cartilage tissue provokes strain- and diffusion-generated electric potentials. Degeneration of the cartilage matrix results in a loss of this fixed microenvironment, leading to the disruption of the physiological electric field which is important for tissue homeostasis (19).

Although therapeutic devices for electric stimulation (ES) of bone are entering the clinical market (1), there are only few studies dealing with ES of cartilage in vitro (19-21). Most of the in vivo and in vitro approaches are based on pulsed electromagnetic fields (PEMF). Data from clinical trials suggest that PEMF are able to improve clinical scores and joint function in osteoarthritic patients (22). In vitro, it was found that PEMF increase the proteoglycan release of alginate-encapsulated CHs (23) and in OA cartilage explants (24). Moreover, Brighton et al developed an experimental setup for capacitive coupled ES in vitro (19-21). In contrast to PEMF, higher frequencies up to $60 \mathrm{kHz}$ are applied on cell cultures and cartilage explants enhancing the synthesis rates of collagen type II and aggrecan $(20,21)$.

To investigate the influence of alternating current (AC) on cellular differentiation process, the aim of the present study was to develop an in vitro test setup for application of electric fields. The electrode design is based on previously published bone stimulation system, enabling direct coupling of $\mathrm{AC}$ and application of defined electric field $(25,26)$. Using the in vitro test setup, we intended to analyze the effects of ES on hyaline-like differentiation of human $\mathrm{CHs}$, mesenchymal stem cells derived from human BM-MSC as well as a co-culture of both cell types. We assumed that ES of cartilage cells has an impact on chondrogenic differentiation and cartilage tissue regeneration. The evaluation of ES effects on activation of $\mathrm{CHs}$ and BM-MSCs contributes to fundamental knowledge as a basis for future applications of ES to improve cartilage healing.

\section{Materials and methods}

Test system for ES. To investigate the influence of electric fields on human $\mathrm{CHs}$ and mesenchymal stem cells, an in vitro setup was developed following the construction of an ES system established by our working group (26). For better handling, the electrode setup was adapted to the dimension of a 6-well plate (Fig. 1A). The cylindrical electrodes (length: $14 \mathrm{~mm}$, diameter: $5 \mathrm{~mm}$ ) were made of pure titanium and separated by a $5 \mathrm{~mm}$-long insulator made of polyetherether ketone (PEEK). The electrode holders were made of PEEK as well and warranted a gap of $3 \mathrm{~mm}$ between electrodes and well bottom. Thus, the cells could be cultured onto a scaffold below the electrodes (Fig. 1B). The lid of the 6-well plate had pre-drilled holes for the contact rods (length: $35 \mathrm{~mm}$; titanium).

The electric potentials inside the stimulation chamber were measured as $\mathrm{V}_{\mathrm{RMS}}$ at defined coordinates at the well bottom using a DC-free sine wave $\left(1 \mathrm{kHz}, 0.7 \mathrm{~V}_{\mathrm{RMS}}\right)$. The values were used to optimize the numerical simulation performed with the finite element method software Comsol Multiphysics (Comsol Multiphysics 5.2; COMSOL, Stockholm, Sweden). Based on these data, distribution of the electric potentials (Fig. 1C, left panel) and electric field norm (Fig. 1C, right panel) within the stimulation chamber was calculated by Comsol Multiphysics, indicating that the cells were stimulated by the electric field with field strengths of $20-35 \mathrm{~V} / \mathrm{m}$.

Cell culture. For the stimulation experiments, human $\mathrm{CHs}$ and mesenchymal stem cells were used. For $\mathrm{CH}$ isolation, human articular knee cartilage were obtained post-mortally from human donors $(n=7$, male $(n=6)$ : $51 \pm 21$ years; female $(\mathrm{n}=1)$ : 52 years) within the first $72 \mathrm{~h}$ after the donor's death. The patients' anamneses and cause of death were unknown. All tissue samples macroscopically showed no indication of OA or degeneration. The study was approved by the Local Ethical Committee of the University of Rostock (registration no. A2011-138). Due to forensic and legal reasons no written approval could be obtained from the patients. However, all issued information relating to the patients in the study are fully anonymized. Human CHs were isolated from the human knee cartilage under sterile conditions as previously described (27) and cultured in Dulbecco's modified Eagle's medium (DMEM; Invitrogen; Thermo Fisher Scientific, Inc., Waltham, MA, USA) containing 10\% fetal calf serum (FCS), $1 \%$ amphotericin $\mathrm{B}, 1 \%$ penicillin-streptomycin and ascorbic acid (50 $\mu \mathrm{g} / \mathrm{ml}$; Sigma-Aldrich; Merck KGaA, Darmstadt, Germany) at $37^{\circ} \mathrm{C}$ under $5 \% \mathrm{CO}_{2}$ and $5 \% \mathrm{O}_{2}$ (hypoxia). The reduced oxygen level was used to mimic the low oxygen partial pressure within cartilage tissue (28). Human $\mathrm{CHs}$ have to be cultured up to the 2nd passage to achieve a sufficient cell number for the experiments. Therefore, cells in passage three were used for electric stimulation.

Human BM-MSCs were obtained from ATCC ${ }^{\circledR}$ (Lot-no. 63208778; LGC Standards, Wesel, Germany) and cultured in the recommended cell culture medium (Mesenchymal Stem Cell Basal Medium; ATCC, Wesel, Germany), supplemented with the suggested growth kit (Mesenchymal Stem Cell Growth kit for BM-MSCs; ATCC) under standard cell culture conditions $\left(37^{\circ} \mathrm{C}, 5 \% \mathrm{CO}_{2}\right.$, $21 \% \mathrm{O}_{2}$ ). As mentioned by Bentivegna et al (29), BM-MSCs should be used for chondrogenic differentiation in vitro between passage three and six, because number of culture passages strictly influences this type of differentiation capacity. Therefore, we cultured BM-MSCs up to the 3rd passage to achieve a sufficient cell number for all the experiments. BM-MSCs were used in passage four for the experiments. 

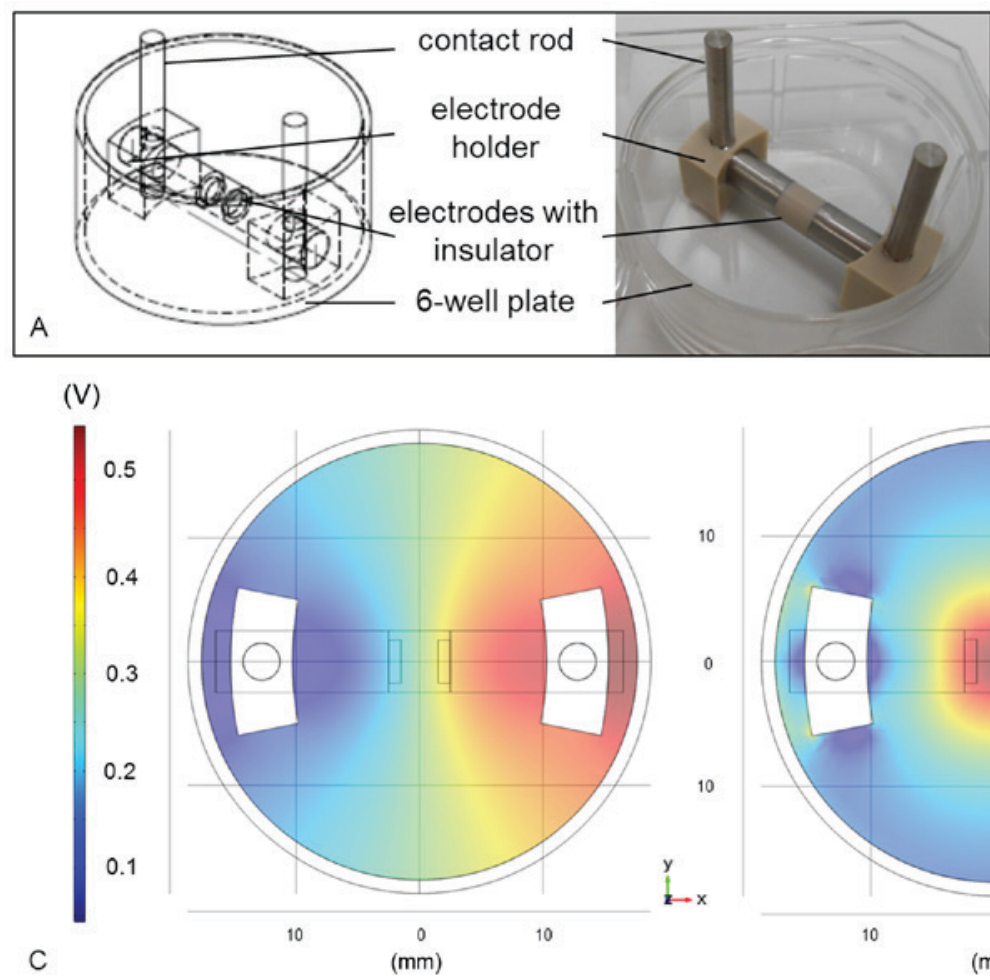

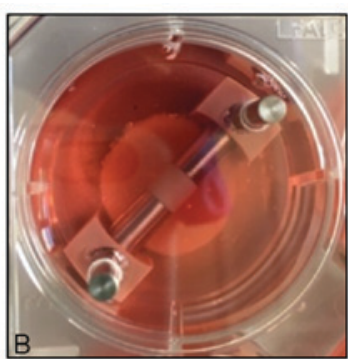

ind

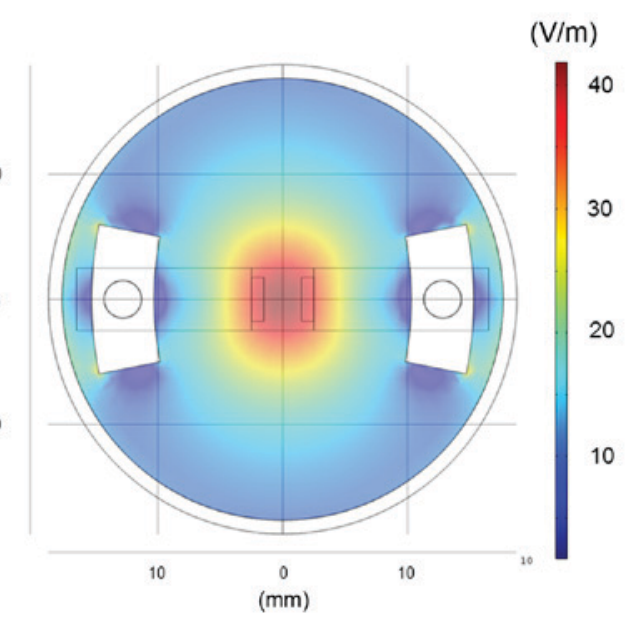

Figure 1. Overview of the in vitro test setup. (A) Three-dimensional technical drawing and image of the stimulation chamber. (B) Human chondrocytes and/or mesenchymal stem cells were cultured onto a collagen-based scaffold and were placed between electrodes and the well bottom. (C) Distribution of electric potential in V (left panel) and field norm in V/m (right panel) simulated by finite element method. The color-coding of the scale bar identifies the range of values for either electric potential (V) or field norm (V/m).

Cell experiments. For the ES experiments, the CHs or BM-MSCs were seeded onto a bovine collagen-based scaffold with a diameter of $20 \mathrm{~mm}\left(1 \times 10^{5}\right.$ cells $\left./ \mathrm{cm}^{2}\right)$. For the co-culture experiments, the cells (CHs and BM-MSCs) were mixed at a ratio of 1:2 prior to cell seeding. The used matrix is a two-layered, bioresorbable scaffold made of collagen type I (MedSkin Solutions Dr. Suwelack AG, Billerbeck, Germany). Before cell seeding, the scaffolds were stuck to the well bottom in the middle of the well using a biocompatible silicon glue (Korasilon paste; Kurt Obermeier GmbH \& Co. KG, Bad Berleburg, Germany). The scaffolds were placed precisely under the electrode allowing a reproducible exposure of the cells to the electric field. After adhering for $30 \mathrm{~min}$, the cell/scaffold constructs were overlaid by the cell culture medium DMEM containing $1 \%$ ITS $^{\text {TM }}$ (Insulin-Transferrin-Selenium; BD Biosciences, Franklin Lakes, NJ, USA), ascorbic acid (50 $\mu \mathrm{g} / \mathrm{ml})$, dexamethasone (100 nM; Sigma-Aldrich; Merck KGaA), insulin-like growth factor-1 (IGF-1, $50 \mathrm{ng} / \mathrm{ml}$; R\&D Systems GmbH, Wiesbaden, Germany) as well as transforming growth factor- $\beta 1$ (TGF- $\beta 1$; $50 \mathrm{ng} / \mathrm{ml}$; tebu-bio $\mathrm{GmbH}$, Offenbach, Germany) and were incubated for $72 \mathrm{~h}$ at $37^{\circ} \mathrm{C}$ under $5 \% \mathrm{CO}_{2}$ either at $21 \% \mathrm{O}_{2}$ (normoxia) or $5 \% \mathrm{O}_{2}$ (hypoxia) $(27,30,31)$. Prior to $\mathrm{ES}$, the medium was replaced by the fresh cell culture medium (mentioned above), but without the addition of the growth factors TGF- $\beta 1$ and IGF-1. AC as sinusoidal signal with a frequency of $1 \mathrm{kHz}$ and $0.7 \mathrm{~V}_{\mathrm{RMS}}$ was applied to the stimulation chamber for seven days using a Metrix GX 305 function generator (Metrix Electronics, Hampshire, UK). Cells were stimulated three times per day for $45 \mathrm{~min}$ each, with $225 \mathrm{~min}$ breaks between stimulations. During ES, cells were cultured at $37^{\circ} \mathrm{C}$ under $5 \% \mathrm{CO}_{2}$ and either at 21 or $5 \%$ oxygen level. For unstimulated control, cells were similarly cultured in the presence of the electrode system, however, without connection to the function generator.

Cell biological tests. Metabolic activity following ES was examined by water soluble tetrazolium (WST-1) assay (Takara Bio, Inc., Shiga, Japan) as recommended by the manufacturer. This colorimetric assay is based on the reduction of tetrazolium salt into formazan salt catalyzed by intercellular dehydrogenases. The amount of generated formazan reflects the metabolic activity of the cells. Cell/scaffold constructs were incubated with diluted WST-reagent (1:10 with cell culture medium) for $60 \mathrm{~min}$. Afterwards, the absorbance was measured at $450 \mathrm{~nm}$ (reference: $630 \mathrm{~nm}$ ) using a spectrophotometer (Infinite ${ }^{\circledR} 200$ PRO; TECAN, Maennedorf, Switzerland).

Gene expression analyses. For gene expression analyses, scaffolds were digested by the cartilage tissue-degrading enzyme collagenase A [2\% in Hank's Balanced Salt Solution; (Gibco; Thermo Fisher Scientific, Inc.), Roche, Mannheim, Germany] for $60 \mathrm{~min}$ at $37^{\circ} \mathrm{C}$ (32). The remaining cell suspension was centrifuged at $180 \mathrm{x}$ g and the cell pellets were resuspended in TriReagent ${ }^{\circledR}$ (Zymo Research, Freiburg, Germany). According to the manufacturer's protocol, the total RNA was extracted by using the Direct-zol RNA MiniPrep kit (Zymo Research), and the RNA concentration was determined by a spectrophotometer (Infinite 200 PRO; TECAN). Single-stranded cDNA was synthesized from total RNA using the High Capacity cDNA Reverse Transcription Kit following the manufacturer's 
instructions (Applied Biosystems; Thermo Fisher Scientific, Inc.). Reverse transcription-quantitative polymerase chain reaction (RT-qPCR) for collagen type I (Forward: 5'-ACG AAGACATCCCACCAATC-3', Reverse: 5'-AGATCACGT CATCGCACAAC-3'), collagen type II (Forward: 5'-ATCCCC TTCGGAGAGTGCTG-3', Reverse: 5'-CCTTTCTGTCCC TTTGGTCCTG-3'), aggrecan (Forward: 5'-ACAAGGTCT CACTGCCCAAC-3', Reverse: 5'-AATGGAACACGATGC CTTTC-3'), SOX9 (Forward: 5'-AGTACCCGCACCTGCACA AC-3', Reverse: 5'-CGCTTCTCGCTCTCGTTCAG-3') and alkaline phosphatase (Forward: 5'-CATTGTGACCACCAC GAGAG-3', Reverse: 5'-CCATGATCACGTCAATGTCC-3') were performed using the innuMIX qPCR MasterMixSyGreen (Analytik Jena AG, Jena, Germany). The cycling conditions for amplification were $95^{\circ} \mathrm{C}$ for $2 \mathrm{~min}, 40$ cycles of $95^{\circ} \mathrm{C}$ for $5 \mathrm{sec}$ and $65^{\circ} \mathrm{C}$ for $25 \mathrm{sec}$. The expression of all genes was normalized to the expression of the corresponding housekeeping gene hypoxanthine guanine phosphoribosyltransferase (HPRT, Forward: 5'-CCCTGGCGTCGTGATTAGTG-3', Reverse: 5'-TCGAGCAAGACGTTCAGTCC-3') and analyzed by $2^{-\Delta \Delta C q}$ method, as previously described $(25,33)$.

Quantification of ECM components. After ES over seven days, supernatants were collected and stored at $-20^{\circ} \mathrm{C}$. The amount of soluble pro-collagen type I (CICP, MicroVue ${ }^{\mathrm{TM}}$ CICP EIA; QUIDEL Corporation, San Diego, CA, USA) and pro-collagen type II (CPII; IBEX Pharmaceuticals, Québec, Canada) within the cell supernatant was determined using enzyme-linked immunosorbent assays (ELISA). The quantity of the C-terminal pro-peptides of both pro-collagen types correlate with the synthesis rate of mature collagen, because the pro-peptides are cleaved from the collagen molecules during incorporation into extracellular collagen fibrils $(34,35)$. Both ELISA assays were conducted according to manufacturer's specifications. Absorbance was measured at a wavelength of $450 \mathrm{~nm}$ for CPII and $405 \mathrm{~nm}$ for CICP using the Opsys MR microplate reader (Dynex Technologies, Denkendorf, Germany).

The glycosaminoglycan (GAG) content was measured in the sample supernatant by Blyscan ${ }^{\mathrm{TM}}$ GAG assay (Biocolor Pharmaceuticals, Inc., Carrickfergus, UK). It quantifies the sulphated proteoglycans and GAGs using 1,9-dimethylmethylene blue. Samples were digested by papain $(20$ units $/ \mathrm{mg}$ in $0.2 \mathrm{M}$ sodium phosphate buffer, $\mathrm{pH}$ 6.4; Sigma-Aldrich; Merck KGaA) overnight at $65^{\circ} \mathrm{C}$. Afterwards, the assay was performed following manufacturer's instructions and absorbance was measured at $656 \mathrm{~nm}$ using a spectrophotometer (Infinite ${ }^{\circledR} 200$ PRO; TECAN).

Additionally, the total protein content of samples was quantified using Qubit ${ }^{\circledR}$ protein assay (Thermo Fisher Scientific, Inc.). Afterwards, collagen or GAGs contents were normalized to total protein amount.

Data illustration and statistical analysis. Data are presented as box plots, whereby the boxes identify the upper and lower percentile, the horizontal lines within the boxes indicate the median and the whiskers denote minimum and maximum values. For all analyses a minimum of three independent donors were used. Since the data obtained were assumed to be not normally distributed, the statistical tests were conducted using the Mann-Whitney $\mathrm{U}$ test and for comparison of more than 2 data sets Kruskal-Wallis test with Dunn-Bonferroni post hoc tests were used (GraphPad Prism 6.0; GraphPad Software, Inc., La Jolla, CA, USA). P $<0.05$ was considered to indicate a statistically significant difference.

\section{Results}

Cell viability following ES. Human CHs and BM-MSCs were cultured onto a collagen-based 3D scaffold either separately or as co-culture (CH+BM-MSCs). The used collagen-based scaffold was stabile over the whole cultivation period and no sign of size reduction was observed. Cells were exposed to an electric field for seven days and afterwards metabolic activity was measured by WST-1 assay (Fig. 2), whereby a high optical density (OD) correlates with a high metabolic activity of the cells. Under normoxia for all three groups metabolic activity of cells exposed to electric field was not affected compared to respective control cells (Fig. 2A). Equally, under hypoxic culture conditions metabolic activity of $\mathrm{CH}$ and CH+BM-MSCs was not influenced by ES (Fig. 2B). Metabolic activity of BM-MSCs cultured under hypoxia and stimulated by ES was non-significantly decreased by $40 \%$ compared to unstimulated control. Nevertheless, the metabolic activity of BM-MSCs was still higher than metabolic activity of the other cells cultured under hypoxic condition.

Metabolic activity of human CH, BM-MSCs and co-culture of both cell types was not significantly influenced following ES which indicates that ES had no cytotoxic effect on cellular metabolism.

Alteration of gene expression pattern following ES. Gene expression in human $\mathrm{CH}$, BM-MSCs and $\mathrm{CH}+\mathrm{BM}-\mathrm{MSCs}$ was analyzed after ES over seven days and presented in Fig. 3. Under hypoxia both $\mathrm{CH}$ and BM-MSCs exhibited increased expression of collagen type I mRNA as a marker of unwanted fibrocartilage compared to unstimulated control. However, under both normoxia and hypoxia co-cultured cells (CH+BM-MSCs) displayed a lower level of collagen type I mRNA expression following ES. Regarding hyaline cartilage marker, the results showed that under hypoxia ES caused increased expression of collagen type II mRNA compared to unstimulated control. For CHs cultured under hypoxia collagen type II mRNA expression was significantly enhanced compared to $\mathrm{CHs}$ cultured under normoxia $(\mathrm{P}=0.0473)$. Additionally, in $\mathrm{CH}$ and $\mathrm{CH}+\mathrm{BM}-\mathrm{MSC}$ s cultured under hypoxia ES caused the highest expression level of aggrecan mRNA. Under normoxia only the BM-MSCs displayed increased expression of aggrecan and collagen type II mRNA following ES. Collagen type II mRNA expression of BM-MSCs was even significantly increased compared to collagen type II expression of $\mathrm{CH}(\mathrm{P}=0.0188)$. The expression of alkaline phosphatase mRNA as hypertrophy marker was amplified in BM-MSCs after ES under hypoxia, however, in co-cultured cells it did not. Cultivation of $\mathrm{CHs}$ under normoxia resulted in significantly increased mRNA expression of alkaline phosphatase $(\mathrm{P}=0.0087)$ compared to hypoxia.

Results demonstrated that ES tended to affect gene expression of important chondrogenic markers, especially under hypoxic culture conditions. However, findings were only preliminary 

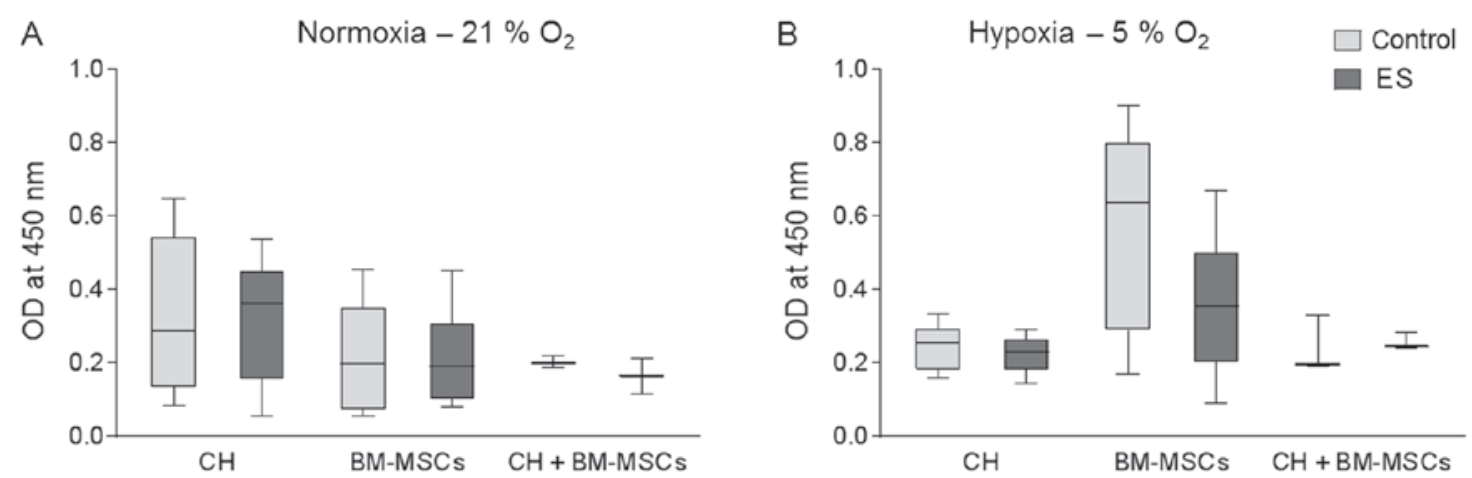

Figure 2. Metabolic activity of CH, BM-MSCs and co-culture of the two (CH+BM-MSCs) following ES under either (A) normoxia or (B) hypoxia. Data of OD were presented as box plots $(\mathrm{CH}, \mathrm{n}=7$; BM-MSCs, $\mathrm{n}=9$; $\mathrm{CH}+\mathrm{BM}-\mathrm{MSCs}, \mathrm{n}=3)$. $\mathrm{CH}$, human chondrocytes; BM-MSCs, bone marrow-mesenchymal stem cells; ES, electric stimulation; OD, optical density.
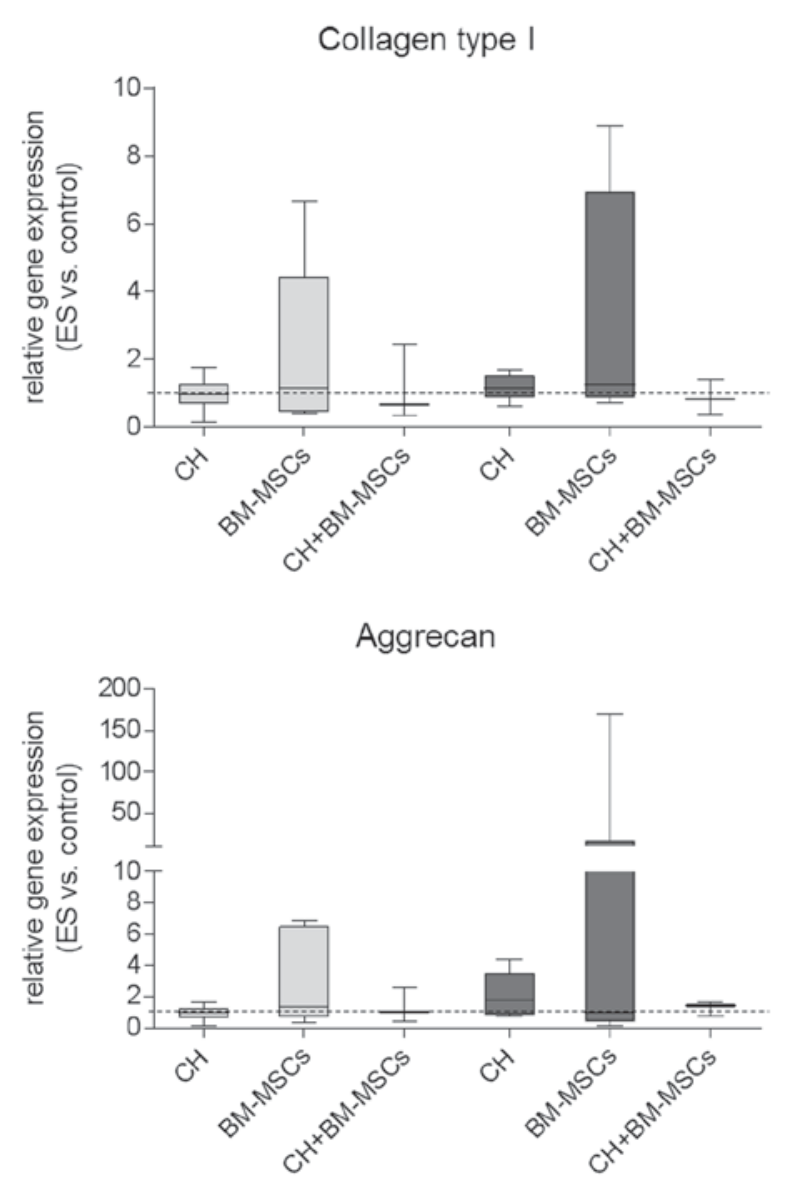
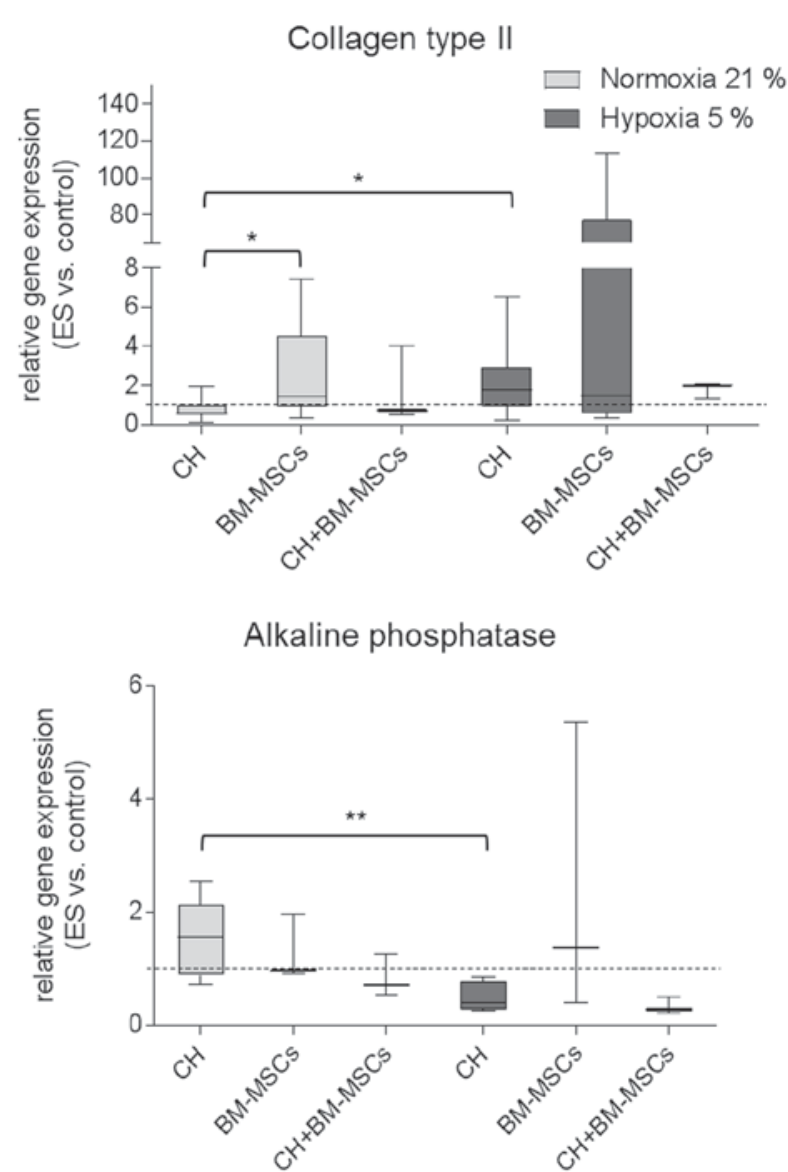

Figure 3. Relative gene expression of $\mathrm{CH}, \mathrm{BM}-\mathrm{MSCs}$ and co-culture of the two $(\mathrm{CH}+\mathrm{BM}-\mathrm{MSCs})$ following ES for seven days under either normoxia (21\% $\left.\mathrm{O}_{2}\right)$ or hypoxia $\left(5 \% \mathrm{O}_{2}\right)$. Data of ES were normalized to unstimulated control and shown as box plots $(\mathrm{CH}, \mathrm{n}=7$; BM-MSCs, $\mathrm{n}=9 ; \mathrm{CH}+\mathrm{BM}-\mathrm{MSCs}, \mathrm{n}=3)$. ${ }^{*} \mathrm{P}<0.05$ and ${ }^{* *} \mathrm{P}<0.01$, as indicated. $\mathrm{CH}$, human chondrocytes; BM-MSCs, bone marrow-mesenchymal stem cells; ES, electric stimulation.

because differences did not reach level of significance probably due to the wide range of the single values in each group.

Synthesis of ECM proteins following ES. The release of collagen type I and II as well as GAGs was measured in the supernatant to investigate the production of ECM proteins following ES (Fig. 4). Under hypoxic culture conditions cells tended to produce more collagen type I than under normoxia, independent of exposition to an electric field. Especially, for unstimulated $\mathrm{CH}$ the collagen type I synthesis is 1.6-fold increased ( $\mathrm{P}=0.0001)$ after cultivation under hypoxia compared to normoxia (Fig. 4A). Simultaneously, the decreased oxygen level of hypoxia supported collagen type II production in $\mathrm{CH}$ (Fig. 4B). For $\mathrm{CH}$, the exposure to an electric field under hypoxia resulted in a significant 1.5-fold higher collagen type II release compared to unstimulated control $(\mathrm{P}=0.0188)$ and also compared to electrically stimulated $\mathrm{CH}$ under normoxia (1.4-fold, $\mathrm{P}=0.04)$. The same development was observed for BM-MSCs, but not for co-culture $\mathrm{CH}+\mathrm{BM}-\mathrm{MSC}$. Under normoxia, collagen type II was not upregulated. 

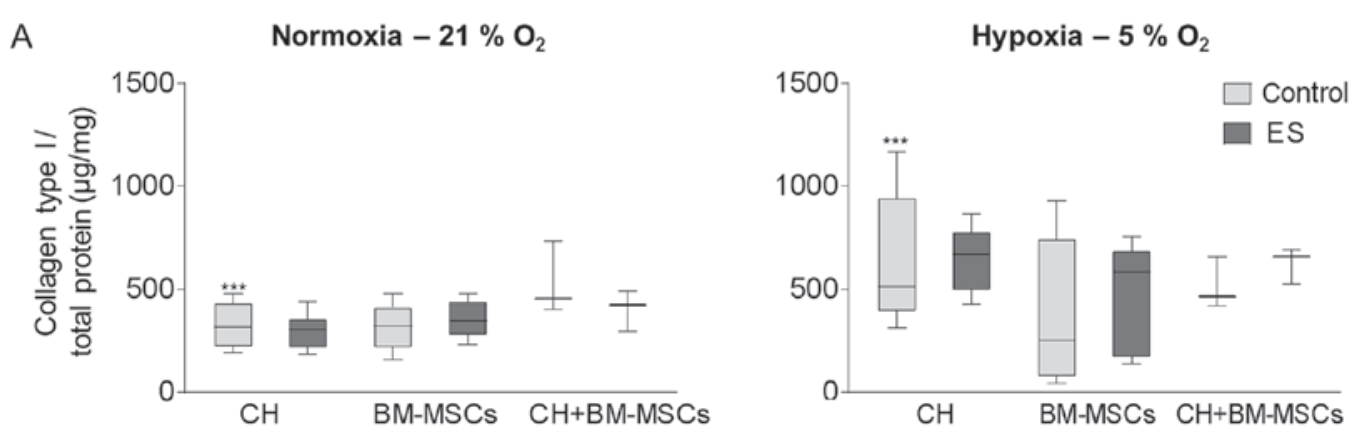

B
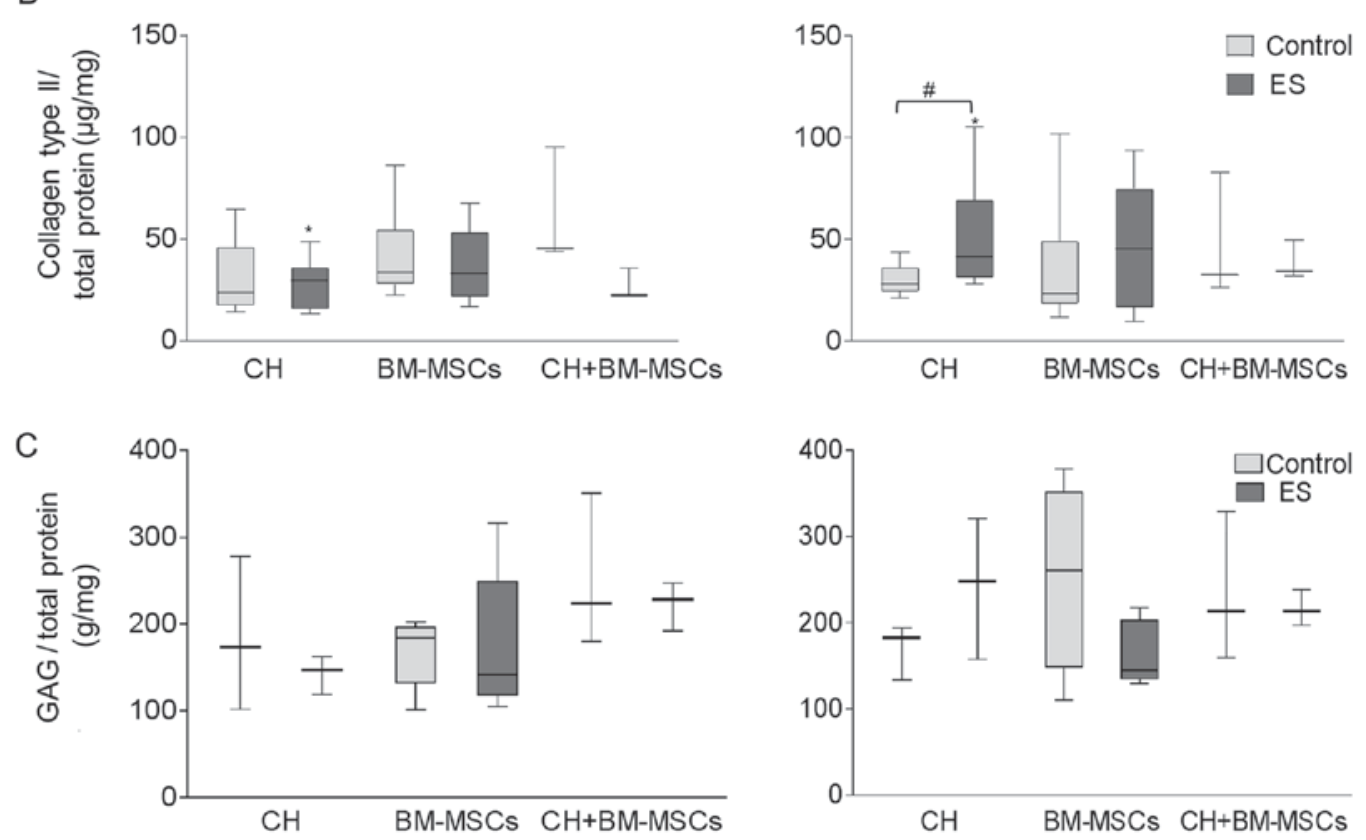

Figure 4. ES of $\mathrm{CH}$, BM-MSCs and co-culture of the two (CH+BM-MSCs) was performed under either normoxia (left panel) or hypoxia (right panel). Following this, the synthesis of (A) collagen types I, (B) collagen type II and (C) GAGs was detected in the supernatant and was related to total protein content. Data were presented as box plots $(\mathrm{CH}, \mathrm{n}=7$; BM-MSCs, $\mathrm{n}=9$; $\mathrm{CH}+\mathrm{BM}-\mathrm{MSCs}, \mathrm{n}=3)$. (A) ${ }^{* * *} \mathrm{P}<0.001$, as indicated for Control normoxia vs. Control hypoxia. (B) ${ }^{*} \mathrm{P}<0.05$, as indicated; " $\mathrm{P}<0.05$, as indicated for ES normoxia vs. ES hypoxia. $\mathrm{CH}$, human chondrocytes; BM-MSCs, bone marrow-mesenchymal stem cells; ES, electric stimulation; GAGs, glycosaminoglycans.

Quantifying the amount of soluble GAGs using Blyscan ${ }^{\mathrm{TM}}$ assay, $\mathrm{CH}$ produced a 1.4-fold increased GAG content following ES under hypoxic culture conditions (Fig. 4C). However, using BM-MSCs a reduced GAG amount was detected following ES compared to unstimulated control under both hypoxic and normoxic culture conditions. Although the co-cultured $\mathrm{CH}+\mathrm{BM}-\mathrm{MSC}$ showed in general a high level of GAG synthesis compared to $\mathrm{CH}$ and BM-MSCs, the GAG release was not influenced during ES.

Even if the synthesis of the undesired fibrocartilage marker collagen type I was promoted under hypoxic culture condition, the production of collagen type II, an important cartilage matrix protein, and soluble GAGs were increased in $\mathrm{CH}$ following ES under hypoxia which is a promising result. Additionally, BM-MSCs showed a tendency to increase collagen type II production following ES compared to unstimulated control, however, differences did not reach level of significance.

\section{Discussion}

A major challenge in orthopedic surgery is the limited healing capacity of cartilage tissue after lesion. Cell-based treatment techniques include the isolation and cultivation of $\mathrm{CHs}$ and BM-MSCs. Subsequently, cells were transferred onto a biomaterial and implanted into the defect site. However, during in vitro cultivation $\mathrm{CHs}$ de-differentiate which could result in formation of fibrocartilage after implantation. A major goal of research is the induction of chondrogenesis of $\mathrm{CHs}$ and BM-MSCs in vitro. Besides the application of growth factors and 3D cultivation, a new approach using biophysical stimulation may be useful to enhance chondrogenesis. In the present study, we have introduced an in vitro test setup for the ES of human CHs and BM-MSCs. The application of electric fields imitate endogenous electric signals initiating the process of chondrogenic differentiation (1,36). To investigate this effect, we cultured human $\mathrm{CHs}$ and BM-MSCs either separately or as co-cultures onto a collagen-based scaffold. Exposure with electric field was performed over seven days and afterwards metabolic activity, gene expression and synthesis of matrix proteins were analyzed.

The construction of the electrode system is based on the design of a stimulation system providing ES on bone cells used in previous studies (26). The electrode system used in the present study supply AC coupled directly to the cells to 
apply a defined electric field. Instead of direct current (DC) stimulation, AC stimulation was chosen, because DC causes additional electrochemical reactions like $\mathrm{pH}$ shift, hydrogen peroxide and formation of reactive oxygen species, which can damage exposed cells (37). The used stimulation parameters like voltage, signal form and stimulation period were chosen in accordance with ES stimulation of bone cells (26). However, frequency was selected due to findings from Brighton et al (21) who had shown that higher frequencies provide a stimulating effect on CHs. Therefore, we used a frequency of $1 \mathrm{kHz}$ for stimulating CHs and BM-MSCs instead of $20 \mathrm{~Hz}$ which had been used for ES of human osteoblasts.

The benefit of our approach was that we used a stimulation system which was adapted to a 6-well plate format and therefore easy to handle. Moreover, the experimental set up allowed the incorporation of a collagen-based scaffold between well bottom and electrode. The use of a scaffold is beneficial, because it mimics physiological environment. This 3D cultivation supports chondrogenic differentiation of $\mathrm{CH}$ and BM-MSCs (38). The scaffold used is proven for clinical therapy and showed high biocompatibility. Additionally, investigation of metabolic activity using WST-1 assay detected no cytotoxic effects of exposed electric field.

In the present study, we have found out that exposure to an electric field increased collagen type II synthesis significantly in human $\mathrm{CHs}$ compared to unstimulated cells under hypoxic culture conditions. Other studies have also detected enhanced collagen type II production after electric field exposure using lower frequencies $(1,36)$. However, Brighton et al (21) exposed $\mathrm{CHs}$ to capacitively coupled electric fields using much higher frequencies $(60 \mathrm{kHz})$ resulting in a significant upregulation of GAG production. Our results for gene expression analyses and examination of soluble GAGs following ES showed that human $\mathrm{CHs}$ tended to express more GAGs than unstimulated controls. By applying increased frequencies for stimulation, proteoglycan production may be enhanced which has to be evaluated in further studies.

Using hypoxic culture conditions during cell cultivation creates more physiological conditions due to avascular nature of the cartilage. Jonitz-Heincke et al (28) had reported that human $\mathrm{CHs}$ cultured in spheroid pellets showed superior collagen type II expression under hypoxic culture conditions compared to normoxia. Our results indicated a promoting effect of hypoxia on chondrogenic differentiation of human CHs. Additionally, gene expression of alkaline phosphatase as marker of undesired hypertrophy was significantly decreased after cultivation under hypoxia compared to normoxia, indicating reduced terminal differentiation of cells which reduce quality of formed cartilage after implantation (39).

The evaluation of extracellular matrix protein synthesis showed that the collagen type I expression was on a very high level compared to collagen type II and glycosaminoglykans (GAGs). The enhanced collagen type I production was detected for all three cell types (CHs, BM-MSCs and co-culture) with and without application of an electric field under both normoxia and hypoxia, indicating that the high collagen type I synthesis was probably not caused by ES or oxygen conditions, but rather by in vitro cultivation. High expression rates of collagen type I is characteristic for BM-MSCs (40). For $\mathrm{CHs}$ this indicates a de-differentiated cell status (41). Since we have determined enhanced collagen type I expression levels either in control or electrical stimulated cells we suspect that the stimulation period was too short to initiate hyaline-like differentiation processes. Previous studies showed that induction of re-differentiation using 3D cultivation in the presence of important growth factors, like fibroblast growth factor (FGF)-2, TGF- $\beta 1-3$ and IGF-1 took several weeks to achieve hyaline-like ECM $(31,42)$. It should also be noted that the use of collagen type I-derived scaffolds, which are usually applied for cartilage repair, will not reflect the physiological environment of chondrocytic cells.

MSCs play a key role for the treatment of cartilage regeneration, and therefore, the induction of chondrogenesis in vitro was investigated in different studies. Our findings showed that BM-MSCs tended to increase collagen type II synthesis following ES compared to unstimulated control. However, differences did not reach level of significance. In the present study, the application of growth factors occurred only for initial pre-cultivation prior to ES to investigate the influence of electric field on chondrogenesis. Although, the essential importance of chondrogenic growth factors for the effect of ES on BM-MSCs was described by Mayer-Wagner et al (43). However, Kwon et al (44) found out that BM-MSCs cultured in micromass cultures showed an upregulation of chondrogenic differentiation markers following ES also in the absence of growth factors. Micromass cultures are a scaffold-free cultivation system in which cells form dense aggregates identical to those in the pre-cartilage environment $(45,46)$. Therefore, micromass cultures could be a key tool for induction of chondrogenesis and may be superior compared to artificial collagen type I scaffolds. For further imitating physiological in vivo conditions, a biofunctionalized scaffold could be integrated within the electrode system in which growth factors are incorporated $(38,47)$. This could enhance the biological response of the BM-MSCs regarding ES.

Different studies have described the promoting effect of co-cultivation of $\mathrm{CHs}$ and BM-MSCs on chondrogenesis (15-17). Especially, regarding results of gene expression analyses co-cultured cells displayed enhanced expression of collagen type II and aggrecan mRNA following ES compared to unstimulated control and single cultivation of BM-MSCs. However, response was inferior compared to single cultivation of CHs. Regarding alkaline phosphatase as a hypertrophic marker, expression levels were decreased compared to unstimulated co-cultured cells. Our results suggest that the co-cultivation of different cell types may be an important approach to realize a more physiological condition and has to be investigated in further studies.

After the initial growth factor pre-incubation over three days, ES was performed over seven days. This stimulation period was suitable for analyzing ES effects on $\mathrm{CH}$ and BM-MSCs, however, differences between groups were rather detected by gene expression analyses than on protein level. Using prolonged stimulation period differences in protein synthesis might be detected more precisely. Furthermore, results of gene expression showed high inter-individual ranges of single values, which probably was a consequence of high donor variability and low sample number impairing achievement of significance level. Additionally, for gene expression analysis, we used the whole scaffold for RNA isolation und subsequent reverse transcription. Consequently, the relative 
gene expression rates of the investigated differentiation marker represent mean values of the cells distributed on the whole scaffold and exposed to the entire range of field strengths. This could also be a reason for high range of single gene expression values. In further studies we will analyze the cellular response within the respective electric field norms to find possible differences in gene expression rates depending on intensity of electric field. However, in this first set up, the primary goal of the study was to determine the overall effects of ES on human $\mathrm{CHs}$ and mesenchymal stem cells.

The design of the stimulation chamber is appropriate for the application of electric fields on cells cultured onto a 3D scaffold. This indicates that the presented stimulation system is a valuable tool to investigate the influence of ES on human CHs, BM-MSCs and a co-culture of both. Reduction of oxygen levels during cultivation could enhance cellular chondrogenesis. However, the culture conditions have to be further adapted to the particular needs of each cell type in order to optimize the ES parameters for $\mathrm{ES}$ of $\mathrm{CHs}$ and BM-MSCs.

\section{Acknowledgements}

The authors would like to thank Mrs. Doris Hansmann and Ms. Anika Witt (Department of Orthopaedics, Restock University Medical Centre, Rostock, Germany) for technical support, as well as Professor Andreas Büttner and Dr. Diana Boy (Institute of Forensic Medicine, Rostock University Medical Centre, Rostock, Germany) for providing the post-mortally obtained knee cartilage.

\section{Funding}

The present study was funded by the AFOR foundation and the German Research Foundation (DFG) via the GRK Welisa.

\section{Availability of data and materials}

The data used and analyzed during the current study are available from the corresponding author on reasonable request.

\section{Authors' contributions}

RB, AJH, JZ, TB and BH designed the study, and TB and JZ designed and validated the stimulation chamber used. $\mathrm{BH}$, MK and SK performed the cell experiments and analyzed the data. $\mathrm{BH}, \mathrm{RB}, \mathrm{AJH}, \mathrm{MK}$ and $\mathrm{SK}$ were involved in data interpretation, and $\mathrm{BH}$ and $\mathrm{AJH}$ wrote the manuscript. All authors edited and reviewed the manuscript.

\section{Ethics approval and consent to participate}

The present study was approved by the Local Ethical Committee of the University of Rostock (registration no. A2011-138). As human tissues were obtained post-mortally, for forensic and legal reasons, written approval was not obtained from the patients, which was approved by the local ethical committee; all information relating to the patients has been fully anonymized.

\section{Patient consent for publication}

Not applicable.

\section{Competing interests}

The authors declare that they have no competing interests.

\section{References}

1. Jahr H, Matta C and Mobasheri A: Physicochemical and biomechanical stimuli in cell-based articular cartilage repair. Curr Rheumatol Rep 17: 22, 2015.

2. Rudert M and Wirth CJ: Knorpelregeneration und Knorpelersatz. In: Kompendium der praktischen Medizin. Springer-Verlag Berlin Heidelberg, pp1205-1218, 2000.

3. Brady MA, Waldman SD and Ethier CR: The application of multiple biophysical cues to engineer functional neocartilage for treatment of osteoarthritis. Part II: Signal transduction. Tissue Eng Part B Rev 21: 20-33, 2015.

4. Bernhard JC and Vunjak-Novakovic G: Should we use cells, biomaterials, or tissue engineering for cartilage regeneration? Stem Cell Res Ther 7: 56, 2016.

5. Welsch GH, Mamisch TC, Zak L, Blanke M, Olk A, Marlovits S and Trattnig S: Evaluation of cartilage repair tissue after matrix-associated autologous chondrocyte transplantation using a hyaluronic-based or a collagen-based scaffold with morphological MOCART scoring and biochemical T2 mapping: Preliminary results. Am J Sports Med 38: 934-942, 2010.

6. Pasold J, Zander K, Heskamp B, Grüttner C, Lüthen F, Tischer T, Jonitz-Heincke A and Bader R: Positive impact of IGF-1-coupled nanoparticles on the differentiation potential of human chondrocytes cultured on collagen scaffolds. Int J Nanomedicine 10: 1131-1143, 2015.

7. Farrell E, O'Brien FJ, Doyle P, Fischer J, Yannas I, Harley BA, O'Connell B,Prendergast PJ and Campbell VA: A collagen-glycosaminoglycan scaffold supports adult rat mesenchymal stem cell differentiation along osteogenic and chondrogenic routes. Tissue Eng 12: 459-468, 2006.

8. Caldwell KL and Wang J: Cell-based articular cartilage repair: The link between development and regeneration. Osteoarthritis Cartilage 23: 351-362, 2015.

9. Steinert AF, Ghivizzani SC, Rethwilm A, Tuan RS, Evans CH and Nöth U: Major biological obstacles for persistent cell-based regeneration of articular cartilage. Arthritis Res Ther 9: 213, 2007.

10. Ko CK, Lee EY,Jang JD, Kim SJ, Suh DS and Chang CH: Cartilage regeneration using a fibrin and autologous cultured chondrocytes mixture in a canine model. J Tissue Sci Eng 3: 114, 2012.

11. Nukavarapu SP and Dorcemus DL: Osteochondral tissue engineering: Current strategies and challenges. Biotechnol Adv 31: 706-721, 2013.

12. Pittenger MF, Mackay AM, Beck SC, Jaiswal RK, Douglas R, Mosca JD, Moorman MA, Simonetti DW, Craig S and Marshak DR: Multilineage potential of adult human mesenchymal stem cells. Science 284: 143-147, 1999.

13. Huang BJ, Hu JC and Athanasiou KA: Cell-based tissue engineering strategies used in the clinical repair of articular cartilage. Biomaterials 98: 1-22, 2016.

14. Madeira C, Santhagunam A, Salgueiro JB and Cabral J: Advanced cell therapies for articular cartilage regeneration. Trends Biotechnol 33: 35-42, 2015.

15. Liu X, Sun H, Yan D, Zhang L, Lv X, Liu T, Zhang W, Liu W, Cao Y and Zhou G: In vivo ectopic chondrogenesis of BMSCs directed by mature chondrocytes. Biomaterials 31: 9406-9414, 2010.

16. Meretoja VV, Dahlin RL, Kasper FK and Mikos AG: Enhanced chondrogenesis in co-cultures with articular chondrocytes and mesenchymal stem cells. Biomaterials 33: 6362-6369, 2012.

17. Levorson EJ, Santoro M, Kasper FK and Mikos AG: Direct and indirect co-culture of chondrocytes and mesenchymal stem cells for the generation of polymer/extracellular matrix hybrid constructs. Acta Biomater 10: 1824-1835, 2014

18. Heng BC, Cao T and Lee EH: Directing stem cell differentiation into the chondrogenic lineage in vitro. Stem Cells 22: 1152-1167, 2004. 
19. Xu J, Wang W, Clark CC and Brighton CT: Signal transduction in electrically stimulated articular chondrocytes involves translocation of extracellular calcium through voltage-gated channels. Osteoarthritis Cartilage 17: 397-405, 2009.

20. Wang W, Wang Z, Zhang G, Clark CC and Brighton CT: Up-regulation of chondrocyte matrix genes and products by electric fields. Clin Orthop Relat Res (427 Suppl): S163-S173, 2004.

21. Brighton CT, Wang W and Clark CC: The effect of electrical fields on gene and protein expression in human osteoarthritic cartilage explants. J Bone Joint Surg Am 90: 833-848, 2008.

22. Vavken P, Arrich F, Schuhfried O and Dorotka R: Effectiveness of pulsed electromagnetic field therapy in the management of osteoarthritis of the knee: A meta-analysis of randomized controlled trials. J Rehabil Med 41: 406-411, 2009.

23. Fioravanti A, Nerucci F, Collodel G, Markoll R and Marcolongo R: Biochemical and morphological study of human articular chondrocytes cultivated in the presence of pulsed signal therapy. Ann Rheum Dis 61: 1032-1033, 2002.

24. Ongaro A, Pellati A, Masieri FF, Caruso A, Setti S, Cadossi R, Biscione R, Massari L, Fini M and De Mattei M: Chondroprotective effects of pulsed electromagnetic fields on human cartilage explants. Bioelectromagnetics 32: 543-551, 2011

25. Hiemer B, Ziebart J, Jonitz-Heincke A, Grunert PC, Su Y, Hansmann D and Bader R: Magnetically induced electrostimulation of human osteoblasts results in enhanced cell viability and osteogenic differentiation. Int J Mol Med 38: 57-64, 2016.

26. Dauben TJ, Ziebart J, Bender T, Zaatreh S, Kreikemeyer B and Bader R: A novel in vitro system for comparative analyses of bone cells and bacteria under electrical stimulation. Biomed Res Int 2016: 5178640, 2016.

27. Jonitz A, Lochner K, Peters K, Salamon A, Pasold J, Mueller-Hilke B, Hansmann D and Bader R: Differentiation capacity of human chondrocytes embedded in alginate matrix. Connect Tissue Res 52: 503-511, 2011.

28. Jonitz-Heincke A, Klinder A, Boy D, Salamon A, Hansmann D, Pasold J, Buettner A and Bader R: In vitro analysis of the differentiation capacity of postmortally isolated human chondrocytes influenced by different growth factors and oxygen levels. Cartilage: 1947603517719318, 2017

29. Bentivegna A, Roversi G, Riva G, Paoletta L, Redaelli S, Miloso M, Tredici G and Dalprà L: The effect of culture on human bone marrow mesenchymal stem cells: Focus on DNA methylation profiles. Stem Cells Int 2016: 5656701, 2016.

30. Chua KH, Aminuddin BS, Fuzina NH and Ruszymah BH: Insulin-transferrin-selenium prevent human chondrocyte dedifferentiation and promote the formation of high quality tissue engineered human hyaline cartilage. Eur Cell Mater 9: 58-67, 2005

31. Jonitz A, Lochner K, Tischer T, Hansmann D and Bader R: TGF- $\beta 1$ and IGF-1 influence the re-differentiation capacity of human chondrocytes in $3 \mathrm{D}$ pellet cultures in relation to different oxygen concentrations. Int J Mol Med 30: 666-672, 2012.

32. Peterkofsky B: Bacterial collagenase methods in enzymology 82 453-471, 1982.

33. Livak KJ and Schmittgen TD: Analysis of relative gene expression data using real-time quantitative PCR and the 2(-Delta Delta C(T)) method. Methods 25: 402-408, 2001.
34. Saggese G, Bertelloni S, Baroncelli GI and Di Nero G: Serum levels of carboxyterminal propeptide of type I procollagen in healthy children from 1st year of life to adulthood and in metabolic bone diseases. Eur J Pediatr 151: 764-768, 1992.

35. Parfitt AM, Simon LS, Villanueva AR and Krane SM: Procollagen type I carboxy-terminal extension peptide in serum as a marker of collagen biosynthesis in bone. Correlation with Iliac bone formation rates and comparison with total alkaline phosphatase. J Bone Miner Res 2: 427-436, 1987.

36. Vaca-GonzálezJJ, Guevara JM, VegaJF andGarzón-Alvarado DA: An in vitro chondrocyte electrical stimulation framework: A methodology to calculate electric fields and modulate proliferation, cell death and glycosaminoglycan synthesis. Cel Mol Bioeng 9: 116-126, 2016.

37. Windisch C, Kolb W, Röhner E, Wagner M, Roth A, Matziolis G and Wagner A: Invasive electromagnetic field treatment in osteonecrosis of the femoral head: A prospective cohort study. Open Orthop J 8: 125-129, 2014.

38. Vinatier C, Mrugala D, Jorgensen C, Guicheux J and Noël D: Cartilage engineering: A crucial combination of cells, biomaterials and biofactors. Trends Biotechnol 27: 307-314, 2009.

39. Chen S, Fu P, Cong R, Wu H and Pei M: Strategies to minimize hypertrophy in cartilage engineering and regeneration. Genes Dis 2: 76-95, 2015.

40. Lefebvre V and Smits P: Transcriptional control of chondrocyte fate and differentiation. Birth Defects Res C Embryo Today 75: 200-212, 2005.

41. Sliogeryte K, Botto L, Lee DA and Knight MM: Chondrocyte dedifferentiation increases cell stiffness by strengthening membrane-actin adhesion. Osteoarthritis Cartilage 24: 912-920, 2016.

42. Haleem AM and Chu CR: Advances in tissue engineering techniques for articular cartilage repair. Oper Tech Orthop 20: 76-89, 2010.

43. Mayer-Wagner S, Passberger A, Sievers B, Aigner J, Summer B, Schiergens TS, Jansson V and Müller PE: Effects of low frequency electromagnetic fields on the chondrogenic differentiation of human mesenchymal stem cells. Bioelectromagnetics 32: 283-290, 2011.

44. Kwon HJ, Lee GS and Chun H: Electrical stimulation drives chondrogenesis of mesenchymal stem cells in the absence of exogenous growth factors. Sci Rep 6: 39302, 2016.

45. Yoon HH, Bhang SH, Shin JY, Shin J and Kim BS: Enhanced cartilage formation via three-dimensional cell engineering of human adipose-derived stem cells. Tissue Eng Part A 18 1949-1956, 2012.

46. Nydegger UE: Progress in complement research: 1984. Year Immunol: 171-174, 1985.

47. Chen FH, Rousche KT and Tuan RS: Technology insight: Adult stem cells in cartilage regeneration and tissue engineering. Nat Clin Pract Rheumatol 2: 373-382, 2006.

This work is licensed under a Creative Commons

Attribution-NonCommercial-NoDerivatives 4.0 International (CC BY-NC-ND 4.0) License. 